\section{$\underset{\substack{\text { hommes } \\ \text { \& migrations }}}{ }$}

\section{Hommes \& migrations}

Revue française de référence sur les dynamiques

migratoires

$1309 \mid 2015$

Le 3e âge des migrants

\title{
Quelques enjeux de l'accès aux soins des populations immigrées âgées
}

\section{Estelle d'Halluin}

\section{Q OpenEdition \\ Journals}

\section{Édition électronique}

URL : http://journals.openedition.org/hommesmigrations/3069

DOI : 10.4000/hommesmigrations.3069

ISSN : 2262-3353

\section{Éditeur}

Musée national de l'histoire de l'immigration

\section{Édition imprimée}

Date de publication : 1 janvier 2015

Pagination : $39-46$

ISBN : 978-2-919040-30-8

ISSN : $1142-852 X$

\section{Référence électronique}

Estelle d'Halluin, «Quelques enjeux de l'accès aux soins des populations immigrées âgées », Hommes \& migrations [En ligne], 1309 | 2015, mis en ligne le 01 janvier 2017, consulté le 19 avril 2019. URL: http://journals.openedition.org/hommesmigrations/3069; DOI : 10.4000/hommesmigrations.3069 


\title{
QUELQUES ENJEUX \\ DE L'ACCĖS AUX SOINS \\ DES POPULATIONS \\ IMMIGRÉES ÂGÉES
}

par ESTELLE D'HALLUIN, maître de conférences en sociologie, Centre nantais de sociologie (CENS), université de Nantes.

\author{
Les difficultés d'accès aux soins font des migrants âgés \\ une population particulièrement fragile. Leur défiance à l'égard \\ des soignants n'est cependant pas univoque ni généralisable. \\ Elle dépend de plusieurs facteurs comme le statut administratif, \\ la présence de solidarités familiales et la stabilité du logement. \\ Une enquête dans une permanence d'accès aux soins de santé \\ permet d'aborder le cas peu étudié des primo-arrivants. \\ Chez eux, la connaissance des structures d'accueil joue un rôle \\ déterminant.
}

En France, les immigrés âgés sont longtemps restés dans l'ombre. Plusieurs logiques ont concouru à ce processus d'“invisibilisation" : le travail a longtemps légitimé la présence des immigrés aux yeux des sociétés d'accueil et d'origine, et même de l'immigré, entretenant le mythe d'un retour à l'âge de la retraite ${ }^{1}$ et masquant son processus d'enracinement ${ }^{2}$. À partir des années 1990, la question du vieillissement des immigrés, de leur santé et de leur accès aux soins devient un enjeu des politiques publiques. La santé des immigrés est bien documentée aujourd'hui. Les enquêtes mettent en évidence des disparités entre populations immigrées et non immigrées ${ }^{3}$. Pour les plus de 55 ans, l'état de santé déclaré des femmes immigrées est plus dégradé que celui des hommes ${ }^{4}$ et que celui de la population non immigrée. Il s'agit de comprendre quelles sont les difficultés spécifiques à la condition migrante en matière d'accès aux soins.

Les résultats présentés ici s'appuient sur une revue de la littérature et sur une enquête locale au sein d'une métropole française. Cette dernière repose sur une série de 15 entretiens auprès de personnels soignants, de décideurs politiques, d'acteurs 
associatifs engagés dans le champ de l'hébergement ou de la promotion de la santé des populations migrantes, d'une part, et dans le champ plus général de l'accès aux soins, d'autre part ; et sur une série de 10 entretiens auprès d'immigrés âgés primo-arrivants ; une enquête qualitative sur les raisons du maintien dans le dispositif en cours, à partir de dossiers rendus anonymes au sein d'une permanence d'accès aux soins de santé $(n=35)$.

\section{De la spécificité relative des difficultés d'accès aux soins}

Nombre de difficultés d'accès aux soins rencontrées par des immigrés vieillissants sont davantage liées à l'appartenance à une fraction basse des classes populaires quau statut spécifique de migrant. Une littérature en sciences sociales et de grandes enquêtes de l'Institut national de la statistique et des études économiques (Insee) montrent comment la faible rémunération du travail, la complexité et la discontinuité des carrières engendrent une précarité économique qui affecte la santé et l'accès aux soins ${ }^{5}$. En outre, un vieillissement prématuré et une surmortalité plus élevée frappent la classe ouvrière ${ }^{6}$, notamment celle exerçant les 3D Jobs ("Dirty, Degrading and Dangerous unskilled work"). Les immigrés y sont surreprésentés ${ }^{7}$. Ensuite, au sein du monde ouvrier, une moindre écoute du corps ${ }^{8}$ entraîne de plus faibles pratiques préventives et un recours plus tardif aux soins $^{9}$. Enfin, la méconnaissance ou l'incompréhension de procédures administratives complexes sont susceptibles d'entraîner un moindre accès aux droits. À cela s'ajoutent parfois une évaluation différenciée des risques selon les individus ${ }^{10}$, de possibles attitudes de défiance à l'égard du système biomédical ou des médicaments ${ }^{11}$, des habitudes d'auto-soins ${ }^{12}$ qui sont le produit d'expériences antérieures et des conditions de socialisation. Des enjeux non médicaux régissent plus largement les conduites de soin et les arbitrages en matière de santé ${ }^{13}$

Ces logiques sociales affectent donc les aînés immigrés comme d'autres populations. Pour autant, des difficultés les concernent plus spécifiquement : une mauvaise maîtrise de la langue française pour certains et la faiblesse des ressources en interprétariat en France ${ }^{14}$; la diversité des statuts administratifs, la complexité du droit des étrangers et l'importance des statuts précaires ${ }^{15}$; l'exposition à des traitements différentiels ou à la discrimination liée à l'origine ${ }^{16}$ dans un contexte de montée de la xénophobie ; la fréquence des allers-retours avec le pays d'origine ${ }^{17}$. Sans compter l'exposition à la violence (dans le pays d'origine, durant le parcours d'exil) et les troubles psychologiques qui peuvent en résulter. Ces caractéristiques peuvent entraver, complexifier, l'accès aux soins ou entraîner des ruptures de droits. En 2005, une enquête auprès d'un échantillon d'immigrés vieillissants de l'agglomération nantaise montre l'absence fréquente de complémentaire santé, un manque d'information sur les aides financières de la Caisse primaire d'assurance maladie (CPAM), le renoncement aux soins de spécialistes, le besoin exprimé d'accompagnement pour se faire soigner, la méconnaissance ou la réticence

5. Karine Meslin, Kedhidja Benelhadj, “Présentation des études de l'Asamla”, in Le Vieillissement des populations immigrées, Nantes, Asamla/RésO Villes 2014. 6. Emmanuelle Cambois, Caroline Laborde, Jean-Marie Robine, “'La double peine' des ouvriers : plus d'années d'incapacité au sein d'une vie plus courte", in Population et sociétés, n 441, 2008, pp. 1-4. 7. Adrian Favell, “Immigration, migration et libre circulation dans la construction de l'Europe", in Politique européenne, $n^{\circ}$ 31, 2010, pp. 33-64. 8. Olivier Schwartz, La Vie privée des ouvriers. Hommes et femmes du Nord, Paris, PUF, 1990. 9. Karine Meslin, Kedhidja Benelhadj, op. cit. 10. Antoine Rode, "Non-recours aux soins et autonomie assumée. Récit d'enquête", Observatoire des non-recours aux droits et services (Odenore), document de travail, 2011. 11. Johanne Collin, "Observance et fonctions symboliques du médicament", in Gérontologie et société, n 103, 2002, pp. 141-159. 12. Sylvie Fainzang, L'Automédication ou les mirages de l'autonomie, Paris, PUF, 2012. 13. Cécile Desprès, "Négocier ses besoins dans un univers contraint. Le renoncement aux soins en situation de précarité", in Anthropologie \& Santé, n 6, 2013. 14. Anaïk Pian, "La fabrique de l'interprétariat auprès d'immigrés atteints de cancer : la place des proches en question", in Simon Pennec, François Le Borgue-Uguen, Florence Douguet (dir.) Les Négociations du soin. Les professionnels, les malades et leurs proches, Rennes, PUR, 2014. 15. Didier Fassin, Alain Morice, Catherine Quiminal, Les Lois de l'inhospitalité. Les politiques de l'immigration à l'épreuve des sans-papiers, Paris, La Découverte, 1997. 16. Estelle Carde, "Les discriminations selon l'origine dans l'accès aux soins", in Santé publique, $n^{\circ}$ 19, 2007, pp. 99-109. 17. Anaïk Pian, "De l'accès aux soins aux 'trajectoires du mourir'. Les étrangers atteints de cancer face aux contraintes administratives", in Revue européenne des migrations internationales, n²8, 2012, pp. 101-127. 
à faire appel aux services à domicile ou à l'hébergement en structure pour personnes âgées ${ }^{18}$. Voyons maintenant comment s'incarnent ces difficultés à travers la situation de Salim.

\section{Salim ou le refus de soins}

En 2013, une travailleuse sociale d'un foyer Adoma me parle du cas de Salim. Ancien ouvrier du bâtiment, il vivait d'une petite retraite dans une chambre du foyer. Il ne s'était marié ni ici, ni làbas. Les relations avec sa famille d'origine s'étaient progressivement distendues. Une sœur et un frère vivaient dans le Sud, mais sa dernière visite, il y a quelques années, s'était soldée par une dispute autour d'enjeux financiers. Depuis cinq ans, il n'avait pas quitté la ville. Ses fréquentations s'étaient recentrées sur le foyer. Il ne retournait plus au pays, ne recevait pas de nouvelles. Quand la travailleuse sociale du foyer constate une forte dégradation de son état de santé, elle réalise par la même occasion que Salim ne voyait plus de médecin. Son nom avait même été radié du registre de la Sécurité sociale tant il s'était écoulé de temps depuis sa dernière consultation. Nul ne s'était aperçu de cet éloignement du système de soins. La responsable de la résidence fut alertée par des difficultés croissantes à marcher, une hygiène dégradée. Il fut hospitalisé une première fois pour de graves problèmes circulatoires. Par la suite, il refusa tous les soins.

"Je rentrais dans la chambre, raconte l'intervenante sociale, il disait: 'Non, non merci.' Il ne voulait pas voir les infirmières, il ne voulait pas qu'on le soigne. (...) Il fallait l'hospitaliser... avec un vieillissement et effectivement un peu de délire : on lui 'prenait plus de sang à lui qu'aux Français qui étaient à l'hôpital'. (...) Il ne voulait plus être hospitalisé. Du coup, on a fait des soins à domicile, fait livrer des repas. (...) J'apportais des vêtements, on a essayé d'entretenir, de faire du ménage dans sa chambre, mais il refusait. Par exemple, il n'ouvrait pas la porte au laboratoire qui venait faire sa prise de sang ou aux infirmières et au médecin... On a dû remettre une clé au médecin pour qu'elle puisse entrer sans qu'il ouvre. C'est limite! Ça va parce que c'est quelqu'un que je connaissais bien, qu'on a travaillé ensemble, je savais qu'elle était respectueuse. C'est elle

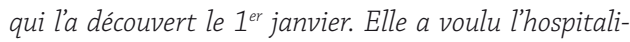
ser, il a refusé en disant : 'Non, jirai lundi.' Elle a dit : 'Lundi, ce sera trop tard!' Elle est revenue le voir. (...) Elle était très inquiète au niveau de sa circulation. Mais il refusait de prendre les médicaments, il refusait les piqûres... Il est mort d'une embolie... Où placer la limite à cette intervention, à cette surveillance ?" (entretien du 13 mai 2013, intervenante sociale en résidence Adoma depuis 2007).

Personne ne s'est manifesté à la déclaration de son décès. Plusieurs mois après, quand deux enfants sans documents officiels ont réclamé ses affaires, il n'y Nombre dedifficultés avait qu'une petite valise.

d'accès aux soins rencontrées Le cas de Salim est margi- pardesimmigrés vieillissants nal mais exemplaire. Margi- sont davantage liées à nal, car la population immigrée installée durablement en France a majoritairement tissé des liens, est "affiliée", voire a accédé à des formes de mobilités sociales et résidentielles. Le regroupement familial a souvent contribué à son enracinement. Même si elle reste bien plus souvent affectée par la précarité, elle recourt aux soins. Pour autant, le cas de Salim est aussi exemplaire d'une frange des immigrés vieillissant en foyer. Là vit une population majoritairement masculine, peu scolarisée, ayant travaillé dans le bâtiment, l'industrie, disposant de revenus modestes ${ }^{19}$. Comme le souligne Choukri Hmed, ils sont souvent peu dotés en ressources économiques, isolés, mais aussi très enracinés au sein de leur foyer, avec des pratiques organisées autour d'un "idéal d'ascétisme et d'abnégation, fruit de leur histoire passée et de leur rapport particulier au travail20". Ils prisent l'espace de vie du 
foyer, pourtant encore souvent inadapté au vieillissement, pour son faible coût, la vie en collectivité, les lieux de sociabilité.

\section{Concilier devoirs des soignants et volontés des patients}

$\mathrm{Au}$ sein du foyer, les cas comme celui de Salim sont plutôt rares. Souvent, les résidents entretiennent une sociabilité dans l'espace du foyer. Ils sont mariés au pays ou y ont de la famille, et nourrissent de ce fait des relations avec la communauté villageoise, notamment par des allers-retours. Leur mobilité est conditionnée par leur état de santé et par les obligations imposées par les réglementations sociales dont dépendent leurs ressources et leur possibilité de se faire soigner. Ils aspirent souvent à une plus grande liberté

Une des missions assignées à la PASS est de faciliter l'accès

à la protection sociale due aux personnes et d'organiser

les soins vers un suivi de proximité de droit commun notamment en orientant le

patient vers le choix d'un médecin traitant hors du dispositif. situation soulève par là-même la problématique des aidants proches dans le recours aux soins. Des réticences existent aussi - et ne sont pas propres aux immigrés - à laisser des professionnels intervenir. Est-ce le refus de se traiter? d'ouvrir la sphère intime de sa chambre ? Les professionnels en sont réduits à des suppositions. Au sein du foyer, "il n'y a pas utilisation optimale des services offerts" : plutôt du nonrecours pour l'aide à la toilette, du recours impulsé avec insistance par le travailleur social pour l'aide à domicile ou le service infirmier... La solidarité entre pairs est privilégiée pour les courses. Le portage de repas est parfois difficile à mettre en place pour des questions pratiques (Comment se servir du microondes ? Comment être là systématiquement à l'heure fixe du rendez-vous ?), de goût, de quantité, parfois de conformité aux prescriptions religieuses. Concernant Salim, travailleurs sociaux et médecins sont restés sur un sentiment d'échec. La question, souligne l'intervenante sociale, "n'est pas une question d'offre, mais de savoir comment la rendre accessible" (ce constat doit être nuancé pour certains services tel que l'interprétariat). Comment anticiper en amont les ruptures de droits et de suivi ? Comment parvenir à rendre effectifs un service de gériatrie volant ou des visites à domicile d'assistantes sociales? Telles sont les réflexions suscitées par cette expérience, où les professionnels ont tenté de concilier la nécessité des soins et le respect des décisions de Salim. Entre une éthique du soin et de l'assistance, d'une part, et l'injonction à promouvoir et à protéger l'autonomie des usagers, d'autre part, ses refus et surtout sa mort ont interrogé les limites de l'accompagnement. Le chibani résidant en foyer incarne bien souvent la figure de l'immigré ayant vieilli en France, même si la plupart vivent en logement individuel21. Mais, au sein de la population immigrée âgée, figurent aussi des personnes plus récemment arrivées en France, dont les conditions d'accès aux soins ont été moins décrites.

\section{Les primo-arrivants âgés : une situation hétérogène}

Les permanences d'accès aux soins de santé (PASS), tout comme les dispensaires associatifs accueillant des populations précaires, sont depuis plusieurs décennies un observatoire des conditions d'accès aux soins. En 2008, une étude auprès des patients de plus de 60 ans d'une PASS montre la fréquence de pathologies athéromateuses, de maladies cardiovasculaires $(75,9 \%)$, de dyslipidémies (53,5\%), de diabète $(13 \%)$ et de cancers $(7,4 \%)^{22}$. Nous nous 
Boîtes aux lettres du foyer Adoma "Les Grésillons".

Gennevilliers, $1^{\text {er }}$ avril 2015 (C) Camille Millerand

sommes fondés sur cette étude afin d'approfondir les déterminants sociaux qui peuvent influencer l'orientation plus ou moins rapide vers un suivi en ville et les modalités de sortie du dispositif. La majorité des patients âgés de plus de 60 ans venus en consultation sont nés à l'étranger. Une minorité réside depuis plus de cinq ans en France. Une majorité est primo-arrivante. D'après l'enquête, les logiques qui conduisent des personnes à migrer à un âge avancé sont plurielles, souvent imbriquées. Les personnes ont évoqué la fuite devant des violences ou des menaces de persécution, leur isolement au pays, l'insistance de leurs enfants ou d'autres proches vivant en France pour les avoir à leurs côtés. La détérioration des conditions de vie, la dégradation de l'état de santé ou un veuvage sont bien souvent les catalyseurs d'une décision prise fréquemment en famille.
Les motifs de la première consultation des patients primo-arrivants sont pluriels : le renouvellement d'un traitement interrompu en raison du voyage (diabète type 2 et hypertension artérielle notamment) et/ou en l'absence de couverture sociale à l'arrivée en France, une demande de conseil médical, des soins de contrôle après une hospitalisation en urgence ou l'apparition de symptômes douloureux (otite, céphalées, malaises, douleurs lombalgiques, abdominales ou mictionnelles...). En cas de symptômes, l'accès à un professionnel de santé est rapide. Une majorité est conduite le jour même (par un proche ou un inconnu) chez un professionnel de santé, voire hospitalisée en urgence. Quand l'enquêté est hébergé par un membre de sa famille, il est souvent examiné par le médecin traitant de celle-ci, les hôtes honorant la consultation et le traitement. Un traitement prolongé trop coûteux peut 
les inciter à se renseigner sur les alternatives possibles. Ils découvrent alors l'existence de la PASS. Dans d'autres cas, le patient est conduit immédiatement aux urgences, à la PASS ou chez Médecins du monde, selon la connaissance des institutions, mais aussi selon l'évaluation par la personne et son entourage de la gravité des symptômes (autodiagnostic). Le type de traitement mis en place dépendra intimement de la pathologie.

\section{Des expériences différenciées}

Une des missions assignées à la PASS est de faciliter l'accès à la protection sociale due aux personnes et d'organiser les soins vers un suivi de proximité de droit commun, notamment en orientant le patient vers le choix d'un médecin traitant hors du dispositif. Or, d'après l'observation de 35 patients de la PASS, des parcours différenciés apparaissent très rapidement. Un tiers bénéficie d’une orientation vers un médecin traitant, tandis que le reste disparaît du dispositif, sans que la continuité des soins ait pu être instituée ou du moins préparée par l'équipe. Les conditions de sortie des PASS se différencient en fonction de la langue, du statut administratif, de la présence de solidarités familiales rapprochées et de la stabilité résidentielle. Les immigrés primo-arrivants peuvent se distribuer différemment en fonction de ces variables. Ils peuvent ou non maîtriser la langue française ou anglaise qui facilite leur parcours de soins, être bénéficiaires ou non d'une couverture médicale. Ils peuvent être dans une situation plus ou moins stable au regard de l'hébergement : les personnes à la rue et en caravane se caractérisent par un "rapport à éclipse ${ }^{23 \text { " à }}$ l'institution. Ils peuvent bénéficier d'une solidarité familiale (aide alimentaire, logement gratuit, traduction, accompagnement aux visites médicales), solidarité d'autant plus rapprochée que les enfants ou les proches aidants sont eux-mêmes résidents de longue durée, francophones, dotés d'un minimum de ressources et d'un logement stable. Leur présence semble ici déterminante dans une sortie du dispositif concertée avec l'équipe soignante. En leur absence, un travailleur social peut jouer ce rôle, notamment pour les personnes hébergées en foyer. Ces différentes variables semblent également peser lourdement sur les autres patients de plus de 60 ans parmi lesquels nous avons resitué les primoarrivants. Elles mériteraient une recherche quantitative plus systématique.

\section{Katinka, en quête d'asile}

Arrêtons-nous sur l'histoire de Katinka, dont la précarité du logement et l'absence de famille fragilisent la continuité des soins. Je la rencontre dans une PASS. Avant de fuir la Russie en 2013, Katinka, 72 ans, n'était jamais venue en France. Elle jouissait en Russie d'"une situation sociale confortable". Elle estime avoir toujours eu "une solide santé" jusqu’à son arrivée en France. Elle ne prenait aucun médicament, ne consultait que très rarement le médecin. Originaire d'Arménie, elle avait, avec son mari, rejoint sa fille en Russie à la fin des années 1990. Vieillissant, ils avaient des difficultés à poursuivre une activité dans le commerce des fruits et légumes. À l'inverse, leur fille avait un "business" florissant dans la capitale russe et besoin d'aide pour garder ses enfants. Après quinze ans de vie en Russie, en 2013, Katinka quitte précipitamment ce pays en raison de violences mafieuses endurées par sa fille. Son mari a trouvé la mort. Acheminée en véhicule par un passeur, elle arrive seule en France, très affaiblie. Le jour de son arrivée, elle est conduite rapidement à la PASS par une personne qui la trouve en état de malaise dans la rue. Elle sera hospitalisée : elle fait une poussée de diabète et sera désormais suivie par la PASS.

Aujourd'hui, l'accès à la couverture médicale universelle est en cours, retardé par une erreur de la CPAM sur son sexe. Elle prend deux fois par jour son traitement pour le diabète. Souvent, elle ne 


\section{// Tableau 1: Premières hypothèses d'après l'enquête exploratoire}

\begin{tabular}{|c|c|c|}
\hline $\begin{array}{c}\text { CARACTÉRISTIQUES } \\
\text { DU PATIENT }\end{array}$ & $\begin{array}{l}\text { INFLUENÇANT UNE SORTIE RAPIDE } \\
\text { ET CONCERTÉE PLUS FRÉQUENTE } \\
\text { VERS UN MÉDECIN GÉNÉRALISTE } \\
\text { LIBÉRAL }\end{array}$ & $\begin{array}{l}\text { INFLUENÇANT UN MAINTIEN } \\
\text { DANS LE SERVICE DE LA PASS } \\
\text { OU UNE SORTIE NON CONCERTÉE }\end{array}$ \\
\hline $\begin{array}{l}\text { LANGUE } \\
\text { (PROPRE AUX IMMIGRÉS) }\end{array}$ & Francophone et anglophone. & Non francophone. \\
\hline $\begin{array}{l}\text { COUVERTURE MÉDICALE } \\
\text { ADOSSÉE À LA RÉGULARITÉ } \\
\text { DU SÉJOUR ET À LA CONDITION } \\
\text { DE RÉSIDENCE (TROIS MOIS } \\
\text { DE PRÉSENCE ANTÉRIEURE) } \\
\text { (PROPRE AUX IMMIGRÉS) }\end{array}$ & $\begin{array}{l}\text { Régime général, couverture } \\
\text { médicale universelle. }\end{array}$ & $\begin{array}{l}\text { Sans couverture médicale } \\
\text { (sous visa touristique, par ex.), } \\
\text { aide médicale d’État. }\end{array}$ \\
\hline $\begin{array}{l}\text { SOLIDARITÉ FAMILIALE } \\
\text { RAPPROCHÉE } \\
\text { (IMMIGRÉS ET NON-IMMIGRÉS) }\end{array}$ & $\begin{array}{l}\text { Présence de membres de la famille } \\
\text { accompagnant l'usager dans ses } \\
\text { démarches. }\end{array}$ & $\begin{array}{l}\text { Absence de membres de la famille } \\
\text { accompagnant l'usager dans } \\
\text { ses démarches. }\end{array}$ \\
\hline $\begin{array}{l}\text { STABILITÉ DE L'HÉBERGEMENT, } \\
\text { QUI CONDITIONNE LE SUIVI } \\
\text { SOCIAL AVEC DES EFFETS } \\
\text { SUR LE PARCOURS DE SOINS } \\
\text { (IMMIGRÉS ET NON-IMMIGRÉS) }\end{array}$ & $\begin{array}{l}\text { Hébergement stable: } \\
\text { logement propre, par la famille } \\
\text { ou un tiers; en institution sur } \\
\text { une longue durée. }\end{array}$ & $\begin{array}{l}\text { Hébergement instable } \\
\text { (temporaire en foyer, à la rue, } \\
\text { chez un tiers). }\end{array}$ \\
\hline
\end{tabular}

sent pas ses pieds en dessous des genoux et tombe. C'est l'une des conséquences du diabète. Elle ne dispose pas d'un hébergement stable, dort parfois à la rue quand le 115 ne trouve pas de place dans les structures d'accueil d'urgence. Elle souhaiterait pouvoir rester durablement dans l'une d'entre elles où elle parvient mieux à s'alimenter et à se reposer, où elle a tissé quelques liens avec le veilleur qui parle russe. Dans d'autres, il n'y a ni repas fourni, ni possibilité de s'allonger pour un temps de repos. La journée, elle reste souvent dans un parc et fréquente aussi avec plaisir l'espace d'activités pour les femmes mis en place localement par le 115. Sa procédure d'asile est en cours. Elle s'inquiète particulièrement de pouvoir stabiliser sa situation pour avoir "un peu de tranquillité pour chercher sa fille, son gendre et ses petits-enfants... et les ramener ici". Katinka espère un territoire de vie moins éclaté, plus adapté à son état de santé, un rapprochement de ses proches, un rapprochement de son lieu de vie et de soins.

\section{Une réponse pluridisciplinaire au cumul des facteurs de vulnérabilité des exilés âgés}

La situation des primo-arrivants présente quelques particularités qui viennent s'associer aux problématiques soulevées antérieurement. Face à de telles 
situations, les professionnels interrogés soulignent l'importance du recours aux interprètes pour articuler les volets des pratiques d'accès aux droits, d'assistance, de prévention et de soins (accès à la couverture sociale, bilan de santé et dépistage, prise en charge médico-psychologique, lien et suivi articulés avec les spécialistes, souvent à l'hôpital, éducation thérapeutique). Aux pratiques de soin, les professionnels ajoutent parfois l'entretien d'espaces de sociabilité, d'échanges et de loisirs, qui peuvent par ailleurs faire défaut aux personnes.

De tels exemples d'accompagnement pluridisciplinaire existent sur le territoire français sous diverses formes : associative (Comité médical pour les exilés en Île-de-France, Case de santé de Toulouse...), consultation publique adossée à l'hôpital (dans certaines PASS), plus rarement en libéral (cabinet recourant à des interprètes)... Dans de tels lieux, les professionnels soulignent la tension entre la nécessité de demeurer un lieu de suivi transitoire, un "sas" vers le droit commun, tout en constatant la nécessité pour certains patients d'un accompagnement régulier et relativement long, quand la structure demeure le seul lieu d'ancrage stable au regard d'une précarité et d'une itinérance résidentielles.

\section{Conclusion}

Salim et Katinka sont loin de résumer la condition des immigrés vieillissant en France. D'une part, l'accent a été mis ici sur un refus de soins et certaines difficultés d'accès aux soins en médecine générale, mais des travaux plus récents montrent la complexité des parcours de soins, notamment pour certaines spécialités, quand viennent se greffer les enjeux du coût de la thérapie dans un contexte de pressions budgétaires et de montée de la figure du migrant-fraudeur ${ }^{24}$. D'autre part, Salim et Katinka incarnent des situations de vulnérabilité sociale auxquelles d'autres échappent ${ }^{25}$. La difficulté est bien ici qu'à décrypter les difficultés d'accès aux soins d'une partie des immigrés âgés, le sociologue risque de contribuer à cette association structurelle relevée par Abdelmalek Sayad à propos de l'immigration: "Ils [les immigrés] n'existent qu'en tant qu'ils posent des problèmes et, à la limite, ils n'existent que par les problèmes qu'ils posent et qui les font exister 26 ." Aussi conviendrait-il de terminer sur le portrait de Ranja. Elle est arrivée en 2013 de Madagascar, soucieuse de vivre auprès des siens et à la demande de deux de ses enfants vivant depuis des décennies en France métropolitaine. En cela, elle reproduit le parcours de sa mère venue vivre ses dernières décennies auprès d'une de ses sœurs. Bien entourée en France, elle organise sa vie entre visites familiales, temps de lecture, marche, pratique religieuse et aide à ses proches (tâches domestiques et garde d'enfants). Divorcée depuis ses 27 ans, ayant élevé trois enfants à Antananarivo grâce à de petits travaux et à la pension alimentaire de son mari, elle a l'habitude de "se débrouiller". Après avoir été aiguillée rapidement à la PASS par Médecins du monde suite à une consultation consécutive à la résurgence de symptômes, le traitement de son hypertension et de la goutte est aujourd'hui stabilisé et sa couverture sociale acquise. Elle se sent bien. Elle sera suivie par le médecin traitant de la famille avec lequel elle dit s'exprimer librement. Aujourd'hui, elle caresse l'espoir de voir son recours contre un refus de réunification familiale aboutir et de s'installer dans un petit appartement près de sa fille : elle garderait ses petits-enfants... Ce sérieux frein administratif levé, elle pourrait incarner la figure d'un accès et d'un parcours de soins sécurisés, tout comme celle d'une expérience de la réunification familiale vécue sereinement. De manière générale, les immigrés âgés posent une question plus universelle pour les sociétés marquées par le vieillissement démographique, celle des enjeux multiples de la réduction des inégalités devant le "bien vieillir", un objectif affiché des politiques publiques. 